\title{
GMR
}

\section{Abnormal metabolites related to bone marrow failure in aplastic anemia patients}

\author{
P. Zhong ${ }^{1}$, J. Zhang ${ }^{2}$ and X. Cui ${ }^{2,3}$ \\ 1'Department of Rehabilitation Medicine, Jinan Military General Hospital, Jinan, China \\ ${ }^{2}$ Department of Hematology, Affiliated Hospital of Shandong University of \\ Traditional Chinese Medicine, Jinan, China \\ ${ }^{3}$ Postdoctoral Research Station of Clinical Medical, Shandong University, Jinan, China
}

Corresponding author: X. Cui

E-mail: cdz45@163.com

Genet. Mol. Res. 14 (4): 13709-13718 (2015)

Received April 16, 2015

Accepted July 26, 2015

Published October 28, 2015

DOI http://dx.doi.org/10.4238/2015.October.28.33

ABSTRACT. Metabolomics is the identification and quantitation of small molecules that are involved in metabolic reactions. Liquid chromatographymass spectrometry (LC-MS) has enjoyed growing popularity as the platform for metabolomic studies due to high throughput, soft ionization, and good coverage of metabolites. Serum metabolites of aplastic anemia (AA) patients and healthy controls were investigated using LC-MS. A wavelet-based method was utilized to find and align LC-MS peaks. Principal component analysis, partial least squares discriminant analysis, and optimized potential for liquid simulations were used to identify differences in metabolite levels, and to reveal useful biomarkers. Thirtytwo metabolites that were significantly altered were detected. Of these metabolites, 23 were successfully identified. In AA patients, metabolites involved amino acid biosynthesis, aminoacyl-tRNA biosynthesis, and ATPbinding cassette transporters were higher than normal, while the levels of metabolites involved in TCA cycles were lower than normal. These 
changes may be the primary cause or result of bone marrow failure in patients with AA.

Key words: Aplastic anemia; Metabolomic; Aminoacyl-tRNA biosynthesis; ABC transporters; Bone marrow failure; Hematopoietic stem cells

\section{INTRODUCTION}

Metabolomics is the scientific study of chemical processes involving metabolites. Specifically, metabolomics is the "systematic study of the unique chemical fingerprints that specific cellular processes leave behind," that is, the study of their small-molecule metabolite profiles (Syggelou et al., 2012). The metabolome represents the collection of all metabolites, which are the end products of cellular processes in a biological cell, tissue, organ, or organism (Jordan et al., 2009). In contrast to genomics, transcriptomics, and proteomics, which address macromolecules with similar chemical properties such as DNA, RNA, and proteins, metabolomics involves the analysis of diverse properties of low-molecular-weight bio-compounds (those less than approximately 1,000 Da). Metabonomics has been identified as an effective tool for biomarker screening (Xue et al., 2008; Bogdanov et al., 2008; Chen et al., 2008), disease diagnosis and prognosis (Claudino et al., 2007; Martínez-Granados et al., 2011; Martínez-Martín et al., 2012), and characterization of a metabolic network (Soga et al., 2003). Metabonomic studies generally employ techniques such as nuclear magnetic resonance spectroscopy, Fourier transform infrared (FT/IR) spectroscopy, and either gas chromatography/mass spectrometry (GC/MS) or liquid chromatography (LC)/MS(Dunn et al., 2005). Compared with GC, LC has lower chromatographic resolution but can potentially measure a wider range of analytes (Gika et al., 2007). Recently, LC has been identified as the ideal tool for screening of metabolites and biomarkers (Wilson et al., 2005).

Aplastic anemia $(A A)$ is a bone marrow failure syndrome, which is characterized by peripheral pancytopenia and marrow hypoplasia. Damage to the bone marrow may be triggered by exposure to environmental compounds, such as chemicals, drugs, viral infections, and possibly endogenous antigens generated by genetically altered bone marrow cells. Although various etiologies and distinct pathophysiological mechanisms may be involved in this process, a unifying feature of most patients with AA is a severe defect in stem cells. As a stem cell disease, AA is very instructive and provides insights into the function and quantity of normal hematopoietic stem cells (HSCs) and their ability to regenerate. As stem cells, HSCs are defined by their ability to replenish all blood cell types (multipotency) and their ability to self-renew. Therefore, HSCs have great potentials in a wide range of clinical applications, and defining the key regulatory genes or biochemical pathways involved at the stem cell level remains an important goal of research for the treatment of blood diseases.

In this study, metabonomic analyses were performed on AA patients to determine the mechanisms that may influence the renewal and proliferation of HSCs in these patients.

\section{MATERIAL AND METHODS}

\section{Patients}

Between September 2010 and February 2013, data from 40 AA patients (23 males and 17 females, median age of 39.95 years; range 11-64 years) were compared with 40 healthy control 
subjects (17 males and 23 females, median age of 38.50 years; range 15-68 years) (Table 1 and Figure 1). These patients were diagnosed at a single institution (Department of Hematology, Affiliated Hospital of Shandong University of Traditional Chinese Medicine). The recruited AA patients had no family history of hematologic disease, and most presented with fatigue and petechiae. Serum samples were collected in the Department of Laboratory Medicine of the hospital. This study was approved by the Institutional Review Board of the Affiliated Hospital of Shandong University of Traditional Chinese Medicine, and written informed consent was obtained from all participants in accordance with the Declaration of Helsinki.

\section{Table 1. Characteristic peaks of Aplastic anemia patient samples.}

\begin{tabular}{ccclcccc}
\hline Peak No. & Retention time & Mass & Name & P value (t-test) & VIP & Fold (A/B) & Change compared with control \\
\hline 1 & 0.581 & 132.0899 & L-Ornithine & 0.000 & 1.220 & 4.745 & $\uparrow$ \\
2 & 0.583 & 146.1055 & L-Lysine & 0.000 & 1.311 & 3.258 & $\uparrow$ \\
3 & 0.655 & 115.0633 & L-Proline & 0.000 & 1.041 & 2.204 & $\uparrow$ \\
4 & 0.676 & 146.0688 & L-Glutamine & 0.000 & 1.248 & 2.850 & $\uparrow$ \\
5 & 0.692 & 192.027 & Citric acid & 0.000 & 1.011 & 2.325 & $\downarrow$ \\
6 & 0.693 & 161.0836 & Indole-3-ethanol & 0.000 & 1.005 & 2.985 & $\uparrow$ \\
8 & 0.720 & 161.1052 & L-Carnitine & 0.000 & 1.220 & 3.144 & $\uparrow$ \\
9 & 0.733 & 181.0736 & L-Tyrosine & 0.000 & 1.077 & 2.735 & $\uparrow$ \\
11 & 0.752 & 113.0588 & Creatinine & 0.000 & 1.012 & 2.354 & $\uparrow$ \\
13 & 1.011 & 149.051 & L-Methionine & 0.000 & 1.389 & 3.521 & $\uparrow$ \\
14 & 1.026 & 117.0794 & L-Valine & 0.000 & 1.286 & 2.631 & $\uparrow$ \\
16 & 1.217 & 131.0958 & L-Leucine & 0.000 & 1.299 & 2.898 & $\uparrow$ \\
18 & 1.989 & 165.0799 & L-Phenylalanine & 0.000 & 1.264 & 2.582 & $\uparrow$ \\
19 & 3.802 & 204.0905 & L-Tryptophan & 0.000 & 1.387 & 3.009 & $\uparrow$ \\
22 & 3.803 & 131.0735 & 3-Methylindole & 0.000 & 1.132 & 2.699 & $\uparrow$ \\
23 & 4.752 & 118.027 & Succinic acid & 0.000 & 1.127 & 1.897 & $\uparrow$ \\
24 & 7.054 & 175.0633 & 3-Indoleacetic acid & 0.000 & 1.026 & 2.923 & $\uparrow$ \\
25 & 8.845 & 175.02 & Isocitric acid & 0.000 & 1.201 & 2.109 & $\uparrow$ \\
27 & 10.151 & 255.2565 & Palmitic amide & 0.000 & 1.360 & 4.912 & $\uparrow$ \\
28 & 11.672 & 379.2494 & Sphingosine-1-phosphate & 0.000 & 1.209 & 1.992 & $\uparrow$ \\
29 & 12.045 & 579.2656 & Taurochenodeoxycholate-3-sulfate & 0.000 & 1.105 & 3.408 & $\uparrow$ \\
31 & 12.439 & 399.3357 & Palmitoyl-L-carnitine & 0.000 & 1.285 & 3.622 & $\uparrow$ \\
32 & 12.668 & 568.3371 & Deoxycholic acid 3-glucuronide & 0.000 & 1.082 & 3.385 & $\uparrow$ \\
\hline
\end{tabular}

\section{A}

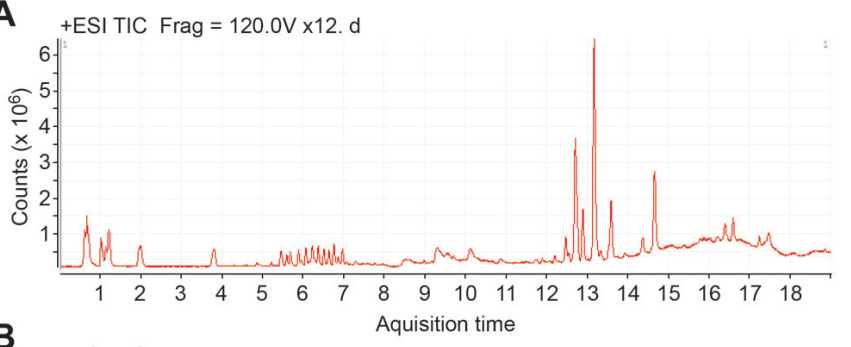

B

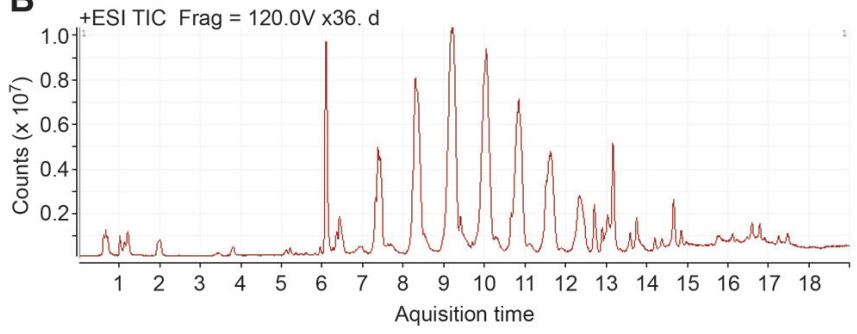

Figure 1. A. Aplastic anemia patient group. B. Normal patient group.. 


\section{Serum specimen pretreatment}

Prior to LC/MS analysis, $300 \mu \mathrm{L}$ acetonitrile was added to the serum samples $(100 \mu \mathrm{L})$, and were vortexed vigorously for $2 \mathrm{~min}$. The sample mixture was left standing for $20 \mathrm{~min}$, followed by $15 \mathrm{~min}$ of centrifugation at $12,000 \mathrm{~g}$ and $4^{\circ} \mathrm{C}$. The supernatant was then dehydrated to complete dryness using a TurboVap nitrogen evaporator (Zymax Corp. USA). Sixty microliters of a mixture of acetonitrile-water $(1: 1, \mathrm{v} / \mathrm{v})$ was added to each of the dried serum extracts, vortex-mixed for 1 $\min$, and subsequently centrifuged at $13,000 \mathrm{~g}$ for $10 \mathrm{~min}$ at $4^{\circ} \mathrm{C}$. Two hundred microliters of the supernatant was then analyzed using LC/MS.

\section{LC separation}

LC analysis was performed using the Agilent 1290 Infinity system (Agilent Technologies, Santa Clara, CA, USA), which consisted of a binary pump, an autosampler, and a UV variable wavelength detector coupled to the Agilent 6120 Single Quad mass detector. Topotecan separation was achieved at $40^{\circ} \mathrm{C}$ using a Discovery HS C 18 column $(100 \mathrm{~mm} \times 2.1 \mathrm{~mm}, 5 \mu \mathrm{m})$ purchased from Supelco Analytical, Bellefonte, PA, USA. The chromatographic data were collected and processed using ChemStation software. The mobile phases comprised of water or methanol mixed with $0.1 \%$ formic acid (solvents $A$ and $B$, respectively). The column was equilibrated and eluted under isocratic conditions with a flow rate of $0.2 \mathrm{~mL} / \mathrm{min}$. The column was maintained at $4^{\circ} \mathrm{C}$, and the injection volume was $5 \mu \mathrm{L}$.

\section{Mass spectrometric assay}

A MS-MS mass spectrometer (Quattro Premier XE, Waters Corp., USA) was used to analyze metabolite ions. Ionization was achieved using electrospray in the positive mode with a spray voltage of $3,500 \mathrm{~V}$. Nitrogen was used as the nebulizer gas and was delivered at a flow rate of $50 \mathrm{~L} / \mathrm{h}$ with a source temperature of $100^{\circ} \mathrm{C}$. The desolvation gas (nitrogen) was heated to $350^{\circ} \mathrm{C}$ and delivered at a flow rate of $600 \mathrm{~L} / \mathrm{h}$. Masses between $\mathrm{m} / \mathrm{z} 100$ and 1,000 were acquired.

\section{Data processing and pattern recognition}

The experimental data of LC/MS were reformed into a matrix including time, ion mass $(\mathrm{m} / \mathrm{z})$, and intensity. Baseline correction and peak finding were performed using the MassHunter software (Version B 03.01, Agilent Technologies). Maximum spectrum of continuous wavelet transform (MSCWT) was used to correct baseline and detect peak positions (Trygg et al., 2007). Peak alignment was performed according to a method previously described in the literature (Smith et al., 2006). The MS analysis system identified each characteristic metabolite corresponding to the featured peak in the Metlin database (http://metlin.scripps.edu) (Smith et al., 2005) and HMDB (http://www.hmdb.ca/) (Wishart et al., 2007). The hit metabolites were verified with textmining techniques.

The targeted profiling data were analyzed using procedures based on principal components analysis (PCA), partial least squares for discriminant analysis (PLS-DA), and optimized potential for liquid simulations (OPLS) after being imported into the SIMCA-P software (Version 11.0). 


\section{RESULTS}

\section{Distinct serum metabonomic profiles of AA patients}

A representative chromatogram from a control group case is shown in Figure 1. Following baseline correction and peak alignment, metabolite signals were obtained. The T-score method was applied to identify featured peaks that were different between AA patients and the control group. All peaks were sorted based on the $\mathrm{p}$ value of Student's $t$-test.

The majority of the peaks visible in the chromatograms were identified according to the NIST (National Institute of Standards and Technology) mass spectra library as endogenous metabolites, which include amino acids, organic acids, carbohydrates, and fatty acids. These metabolites are involved in multiple biochemical processes, particularly in energy and lipid metabolism (Postic et al., 2004). We obtained 32 signals in total. Table 1 shows the 23 specific signals that could be autoidentified using the NIST library by comparing the fragmentation patterns of all fragment ions. The remaining 19 peaks, which could not be identified using the NIST library, are not listed. All amino acids in the "32 metabolites" were further identified by correlating retention times and fragmentation patterns, as well as by comparing them with the corresponding commercially available standards.

Subsequently, the integral values were mean centered for PCA (Figure 2A), PLS-DA (Figure 2B), and OPLS (Figure 2C) using the SIMCA-P+11.0 software package (Umetrics, Umeå, Sweden). PCA and PLS-DA score plots were visualized with the first principal component (t[1]) and the second principal component (t[2]), while OPLS was visualized with the first principal component (t[1]) and the orthogonal component (to[1]). The parameters Q2 (cum) and R2X (cum) were computed to test the validity of the model against overfitting, where R2X (cum) was the total variation explained in the data, and Q2 (cum) was the cross-validated explained variation with increasing reliability as Q2 (cum) approaches 1 (Weljie et al., 2007). The six-fold cross-validation method and permutation test (run 500 times) were performed with the first component to measure the robustness of the model. If Q2 ( $\max$ ), as computed using the permutation test, was less than or equal to Q2 (cum), as calculated using OPLS, then the OPLS model was determined to be robust. Cutoff values with significance levels of $P<0.05$ were used to identify the key variables that were responsible for the discrimination of groups.

The 23 identified metabolites are summarized in Table 1 and include metabolites that are involved in the biosynthesis of amino acids (L-glutamine, L-methionine, L-ornithine, L-tryptophan, L-phenylalanine, L-tyrosine, L-leucine, L-proline, and L-valine), aminoacyl-tRNA biosynthesis (L-glutamine, L-methionine, L-tryptophan, L-phenylalanine, L-tyrosine, L-leucine, L-proline, and L-valine), ATP-binding cassette (ABC) transporters (L-glutamine, L-ornithine, L-phenylalanine, L-leucine, L-proline, and L-valine), as well as those involved in glycolysis and the Krebs cycle (citric acid, succinic acid, and isocitric acid).

\section{Decrease of cellular energy supply obstacles}

In mammalian cells, glucose is the major substrate for ATP production. In normoxic conditions, ATP molecules are mainly produced by glucose metabolism, which consists of the following relay pathways: the oxygen-independent pathway of glucose to pyruvate in cytoplasm, the citric acid cycle (i.e., the Krebs cycle or TCA cycle), and the oxygen-dependent electron transfer chain in the mitochondria. When ATP level was reduced, the major functional components of the 
Krebs cycle including fumarate and malate, were significantly altered. In addition, lactate, the signature metabolite of anaerobic glycolysis, was greatly increased in AA patients. The decrease in energy metabolism may affect the self-renewal and differentiation of HSCs or even trigger caspase activity and eventually HSC apoptosis.

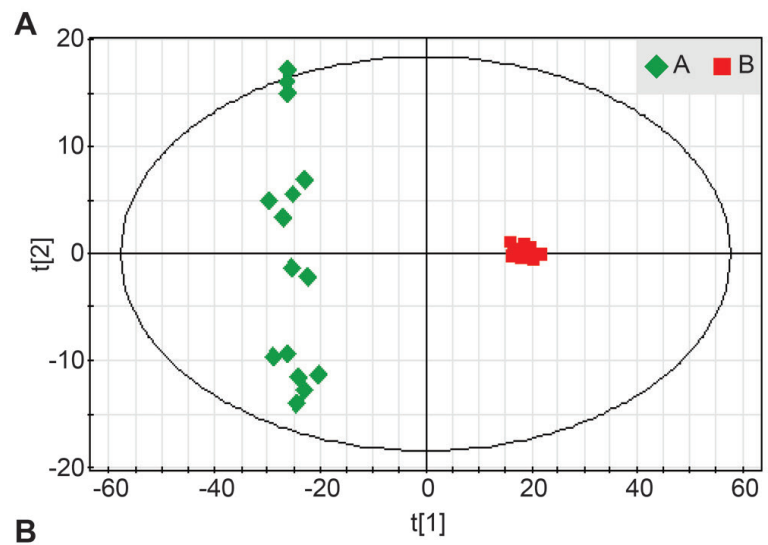

B

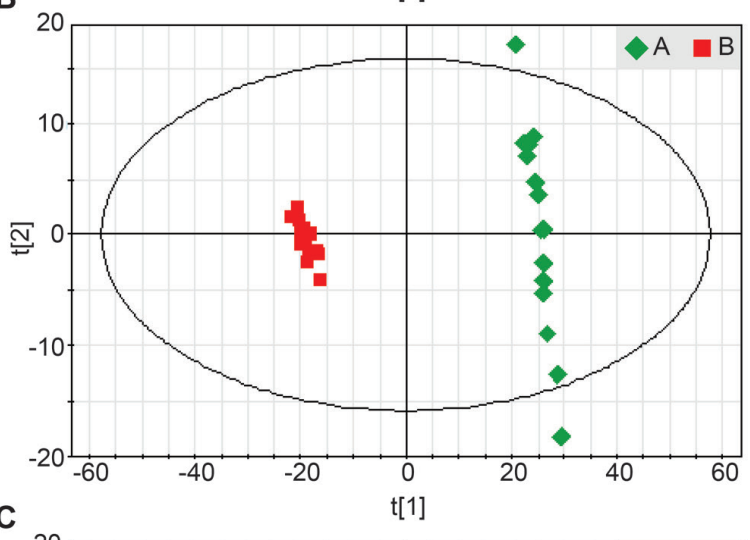

C

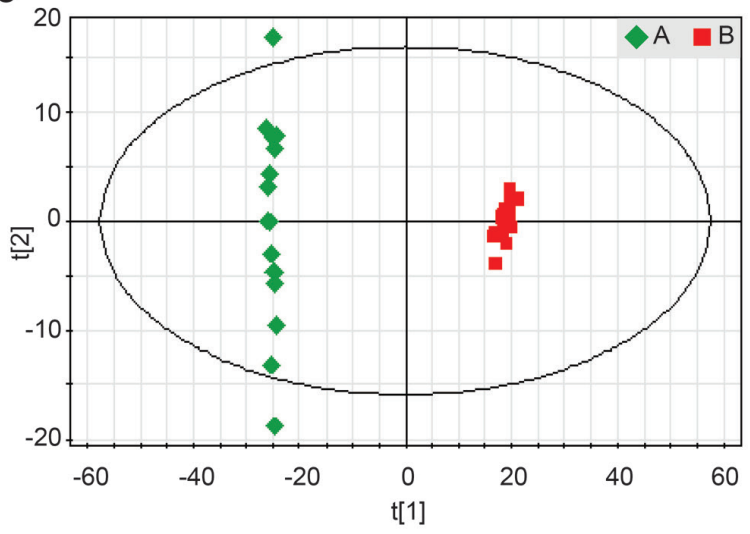

Figure 2. Principal component analysis (PCA) score scatter plot (A), Partial least squares discriminant analysis (PLS-DA) score scatter plot (B), and Optimized potential for liquid simulations (OPLS) score scatter plot (C) for two components, indicating separation between the Aplastic anemia patient group (A) and the normal group (B). 


\section{Disorder of the protein biosynthesis pathway}

Protein biosynthesis occurs when biological cells generate new proteins; it is balanced by the loss of cellular proteins via degradation or export. Translation, the assembly of proteins by the ribosomes, is an essential part of the biosynthetic pathway, along with the synthesis of messenger RNA (mRNA), aminoacylation of transfer RNA (tRNA), co-translational transport, and post-translational modification. Protein biosynthesis is strictly regulated at multiple steps, and also includes error-checking mechanisms. In this study, protein biosynthesis pathways were altered in AA patients, and the metabolites involved in amino acid biosynthesis and aminoacyl-tRNA biosynthesis were increased.

\section{Disorder of the ABC transporter pathway}

Of the 23 identified metabolites, L-glutamine, L-ornithine, L-phenylalanine, L-leucine, L-proline, and L-valine are key metabolites in the $A B C$ transporter pathway. The role of $A B C$ transporter superfamily members in conferring drug resistance has been extensively described, and multidrug resistance-1 (MDR1) and breast cancer resistance protein (BCRP) transporters are expressed in the myeloid lineage in blasts from patients with myeloid or lymphoblastic leukemia (van Grotel et al., 2008; Shman et al., 2011; Qian et al., 2012; Cianfriglia, 2013). Higher levels of $A B C$ transporter pathway metabolites were also observed in AA patients and may play a role in the pathological process responsible for hematopoietic failure.

\section{DISCUSSION}

AA is a bone marrow failure syndrome that is characterized by peripheral pancytopenia and marrow hypoplasia. Damage to the bone marrow may be triggered by environmental exposure, such as exposure to chemicals and drugs; viral infections, and possibly endogenous antigens generated by genetically altered bone marrow cells. Over time, new detection methods have emerged, and bioinformatics has become widely used to collectively analyze diverse biome data. While mRNA gene expression and proteomic analyses may not tell reveal all cellular processes, metabolic profiling can provide an instantaneous snapshot of a cell's physiology. In this study, the metabolic profiles of AA patients were investigated using LC-MS combined with multivariate statistical analysis. When compared with the control group, the levels of L-ornithine, L-proline, L-glutamine, L-tyrosine, creatinine, L-methionine, L-valine, L-leucine, L-phenylalanine, and L-tryptophan were higher in AA patients, whereas the levels of citric acid, succinic acid, and isocitric acid (which are part of glycolysis and the Krebs cycle) were lower.

The lower levels of citric acid, succinic acid, and isocitric acid in AA patients limit the supply of cellular energy, which may disrupt cell signaling, interrupt cellular differentiation, and even induce apoptosis. Furthermore, deficient mitochondrial ATP production resulting from mutations in mitochondrial DNA (mtDNA) may promote chromosomal instability (Gattermann, 2004). Cells with an inadequate ATP supply may have difficulties in the properly segregation of chromosomes during mitosis. These factors may decrease energy metabolism, which can affect the self-renewal and differentiation of HSCs (Zhang and Sadek, 2014). The main site of energy metabolism is the mitochondria, and we recently found (Cui et al., 2012) that mutations of mtDNA may induce apoptosis through the mitochondrial pathway.

Amino acid and aminoacyl-tRNA metabolism were also affected in AA patients. Protein 
synthesis and/or amino acid transport may have been disrupted, which would lead to abnormal consumption of amino acids. Previous studies have indicated that normal amino acid metabolism may protect embryos from oxidative damage (Houghton et al., 2002), and regulate normal intracellular osmotic pressure (Humpherson et al., 2005). Because the levels of amino acid and aminoacyl-tRNA metabolism of AA patients were higher than normal patients, we speculate that disordered amino acid metabolism may damage the balance of intracellular osmotic pressure, and may even affect the self-renewal and differentiation of HSCs. Furthermore, disorders of normal amino acid metabolism may cause HSC apoptosis or death.

The levels of L-glutamine, L-ornithine, L-phenylalanine, L-leucine, L-proline and L-valine in the AA group were higher than in the control group, which reflect disruptions of $A B C$ transporters. $A B C$ transporters are members of one of the largest and oldest protein superfamilies, which is represented in all extant phyla, from prokaryotes to humans (Jones and George, 2004; Kimura et al., 2012). ABC transporters are transmembrane proteins that utilize adenosine triphosphate (ATP) hydrolysis to perform certain biological processes, including the translocation of various substrates across membranes, as well as non-transport-related processes such as the DNA and RNA repair (Costa et al., 2005; Westerterp et al., 2014). MDR1 and BCRP, which belong to the ABC transporter superfamily in the myeloid lineage, are expressed in blasts from patients with acute myeloid leukemia, but only very low to undetectable levels have been measured in normal myelomonocytic cells. The MDR1 gene products P-glycoprotein (P-gp) and BCRP are expressed differentially during hematopoiesis, with the highest levels in primitive bone marrow stem cell populations that are CD34low and CD34-, respectively (van den Heuvel-Eibrink et al., 2007). Therefore, one interpretation is that BCRP expression may define primitive quiescent HSCs, whereas MDR1 may be expressed in more "activated" repopulating HSCs. However, the expression of the Bcrp1/ABCG2 gene is an important determinant of the 'side-population' (SP) phenotype that has been reported in mouse CD34- stem cells (Zhou et al., 2001). In human cells, BCRP also appears to be highly expressed in phenotypically defined populations of primitive HSCs (Scharenberg et al., 2002). Whether there are other transporters that influence self-renewal and differentiation remains to be confirmed, but we suggest that regulation of stem cell physiology by $A B C$ transporters is an important new field of investigation.

Our results indicate that levels of metabolites involved in aminoacyl-tRNA, ABC transporter, and TCA cycle metabolism are different in AA patients as compared to the control group. The underlying mechanism for these differences may be related to metabolomic changes, and which may be the primary cause or result of bone marrow failure in AA. Further studies are needed to elucidate the precise mechanisms responsible for the results observed in this study.

\section{Conflicts of interest}

The authors declare no conflict of interests.

\section{ACKNOWLEDGMENTS}

Research reported in this study was supported by the National Natural Science Foundation of China (\#81202839/H2902), the Natural Science Foundation of Shandong Province, China (\#ZR2012HQ023), the Medical science and technology development Foundation of Shandong Province, China (\#2014WSB23003), the Jinan young star of science and technology plan (\#201406012), and the Affiliated Hospital of Shandong University of Traditional Chinese Medicine and Shandong University, China. 


\section{REFERENCES}

Bogdanov M, Matson WR, Wang L, Matson T, et al. (2008). Metabolomic profiling to develop blood biomarkers for Parkinson's disease. Brain. 131: 389-396.

Chen J, Zhao X, Fritsche J, Yin P, et al. (2008). Practical approach for the identification and isomer elucidation of biomarkers detected in a metabonomic study for the discovery of individuals at risk for diabetes by integrating the chromatographic and mass spectrometric information. Anal. Chem. 80: 1280-1289.

Cianfriglia M (2013). Targeting MDR1-P-glycoprotein (MDR1-Pgp) in immunochemotherapy of acute myeloid leukemia (AML). Ann. Ist. Super. Sanita 49: 190-208.

Claudino WM, Quattrone A, Biganzoli L, Pestrin M, et al. (2007). Metabolomics: available results, current research projects in breast cancer, and future applications. J. Clin. Oncol. 25: 2840-2846.

Costa Cda S, Albuquerque FC, Andrade RV, Oliveira GC, et al. (2005). Transporters in the Paracoccidioides brasiliensis transcriptome: insights on drug resistance. Genet. Mol. Res. 4: 390-408.

Cui X, Lui F, Wang JQ, Zhang WJ, et al. (2012).Complete sequence analysis of mitochondrial DNA of aplastic anemia patients. Gen. Mol. Res.11: 2130-2137.

Dunn WB, Bailey NJ and Johnson HE (2005). Measuring the metabolome: current analytical technologies. Analyst 130: 606-625.

Gattermann N (2004). Mitochondrial DNA mutations in the hematopoietic system. Leukemia 18: 18-22.

Gika HG, Theodoridis GA, Wingate JE and Wilson ID (2007). Within-day reproducibility of an LC-MS-based method for metabonomic analysis: application to human urine. J. Proteome Res. 6: 3291-3303.

Houghton FD, Hawkhead JA, Humpherson PG, Hogg JE, et al. (2002). Non-invasive amino acid turnover predicts human embryo developmental capacity. Hum. Reprod. 17: 999-1005.

Humpherson PG, Leese HJ and Sturmy RG (2005). Amino acid metabolism of the porcine blastocyst. Theriogenology 64: 1852-1866.

Jones PM and George AM (2004). The ABC transporter structure and mechanism: perspectives on recent research. Cell. Mol. Life Sci. 61: 682-699.

Jordan KW, Nordenstam J, Lauwers GY, Rothenberger DA, et al. (2009). Metabolomic characterization of human rectal adenocarcinoma with intact tissue magnetic resonance spectroscopy. Dis. Colon. Rectum 52: 520-525.

Kimura N, Hakamada K, Ikenaga SK, Umehara Y, et al. (2012). Gene expression of ATP-binding cassette transporters during liver regeneration after 90\% hepatectomy in rats. Int. J. Mol. Med. 30: 28-34.

Martínez-Granados B, Morales JM, Rodrigo JM, Del Olmo J, et al. (2011). Metabolic profile of chronic liver disease by NMR spectroscopy of human biopsies. Int. J. Mol. Med. 27: 111-117.

Martínez-Martín N, Blas-García A, Morales JM, Marti-Cabrera M, et al. (2012). Metabolomics of the effect of AMPK activation by AICAR on human umbilical vein endothelial cells. Int. J. Mol. Med. 29: 88-94.

Postic C, Dentin R and Girard J (2004). Role of the liver in the control of carbohydrate and lipid homeostasis. Diabetes Metab. 30: $398-408$.

Qian X, Cao S, Yang G, Dong J, et al. (2012).Variant genotypes of MDR1 C3435T increase the risk of leukemia: evidence from 10 case-control studies. Leuk. Lymphoma 53: 1183-1187.

Scharenberg CW, Harkey MA and Torok-Storb B (2002). The ABCG2 transporter is an efficient Hoechst 33342 efflux pump and is preferentially expressed by immature human hematopoietic progenitors. Blood 99: 507-512.

Shman TV, Fedasenka UU, Savitski VP, Kustanovich AM, et al. (2011). Drug resistance associated properties of blasts subpopulations with different CD34 expression in childhood acute lymphoblastic leukemia (ALL). Exp. Oncol. 33: 145-149.

Smith CA, O'Maille G, Want EJ, Qin C, et al. (2005). METLIN: a metabolite mass spectral database. Ther. Drug Monit. 27: 747-751.

Smith CA, Want EJ, O'Maille G, Abagyan R, et al. (2006). XCMS: processing mass spectrometry data for metabolite profiling using nonlinear peak alignment, matching, and identification. Anal. Chem. 78: 779-787.

Soga T, Ohashi $\mathrm{Y}$, Ueno $\mathrm{Y}$, Naraoka H, et al. (2003). Quantitative metabolome analysis using capillary electrophoresis mass spectrometry. J. Proteome Res. 2: 488-494.

Syggelou A, Lacovidou N, Atzori L, Xanthos T, et al. (2012). Metabolomics in the developing human being. Pediat. Clin. North. Am. 59: 1039-1058.

Trygg J, Holmes E and Lundstedt T (2007). Chemometrics in metabonomics. J. Proteome Res. 6: 469-479.

van den Heuvel-Eibrink MM, van der Holt B, Burnett AK, Knauf WU, et al. (2007). CD34-related coexpression of MDR1 and BCRP indicates a clinically resistant phenotype in patients with acute myeloid leukemia (AML) of older age. Ann. Hematol. 86: 329-337.

van Grotel M, van den Heuvel-Eibrink MM, van Wering ER, van Noesel MM, et al. (2008). CD34 expression is associated with poor survival in pediatric T-cell acute lymphoblastic leukemia. Pediatr. Blood Cancer. 51: 737-740. 
Weljie AM, Dowlatabadi R, Miller BJ, Vogel HJ, et al. (2007). An inflammatory arthritis-associated metabolite biomarker pattern revealed by 1 H NMR spectroscopy. J. Proteome Res. 6: 3456-3464.

Westerterp M, Bochem AE, Yvan-Charvet L, Murphy AJ, et al. (2014). ATP-binding cassette transporters, atherosclerosis, and inflammation. Circ. Res. 114: 157-170.

Wishart DS, Tzur D, Knox C, Eisner R, et al. (2007). HMDB: the Human Metabolome Database. Nucleic Acids Res. 35: D521-526. Wilson ID, Plumb R, Granger J, Major H, et al. (2005). HPLC-MS-based methods for the study of metabonomics. J. Chromatogr. B Analyt. Technol. Biomed. Life Sci. 817: 67-76.

Xue R, Lin Z, Deng C, Dong L, et al. (2008). A serum metabolomic investigation on hepatocellular carcinoma patients by chemical derivatization followed by gas chromatography/mass spectrometry. Rapid Commun. Mass Spectrom. 22: 3061-3068.

Zhang CC and Sadek HA (2014). Hypoxia and metabolic properties of hematopoietic stem cells. Antioxid. Redox Signaling 20: 1891-1901.

Zhou S, Schuetz JD, Bunting KD, Colapietro AM, et al. (2001). The ABC transporter Bcrp1/ABCG2 is expressed in a wide variety of stem cells and is a molecular determinant of the side-population phenotype. Nat. Med. 7: 1028-1034. 